\title{
Wheatstone-Panum limiting case: Occlusion, camouflage, and vergence-induced disparity cues
}

\author{
KOICHI SHIMONO and WA JAMES TAM \\ Communications Research Centre, Ottawa, Ontario, Canada \\ and \\ SACHIO NAKAMIZO \\ Fukuoka University of Education, Fukuoka, Japan
}

\begin{abstract}
We examined effects of binocular occlusion, binocular camouflage, and vergence-induced disparity cues on the perceived depth between two objects when two stimuli are presented to one eye and a single stimulus to the other (Wheatstone-Panum limiting case). The perceived order and magnitude of the depth were examined in two experimental conditions: (1) The stimulus was presented on the temporal side (occlusion condition) and (2) the nasal side (camouflage condition) of the stimulus pair on one retina so as to fuse with the single stimulus on the other retina. In both conditions, the separation between the stimulus pair presented to one eye was systematically varied. Experiment 1 , with 16 observers, showed that the fused object was seen in front of the nonfused object in the occlusion condition and was seen at the same distance as the nonfused object in the camouflage condition. The perceived depth between the two objects was constant and did not depend on the separation of the stimulus pair presented to one eye. Experiment 2, with 45 observers, showed that the disparity induced by vergence mainly determined the perceived depth, and the depth magnitude increased as the separation of the stimulus pair was made wider. The results suggest that (1) occlusion provides depth-order information but not depth-magnitude information, (2) camouflage provides neither depth-order nor depth-magnitude information, and (3) vergence-induced disparity provides both order and magnitude information.
\end{abstract}

One of the most interesting stimulus configurations examined in the field of binocular stereopsis is the Wheatstone-Panum limiting case: ${ }^{1}$ two images are projected on one retina and a single image on the other retina (Panum, 1858/1940; Wheatstone, 1838). The WheatstonePanum limiting case can give rise to an impression of two objects at different depths and is interesting to researchers because, so far, there is no single hypothesis that can adequately account for the percept (Ono, Shimono, \& Shibuta, 1992). For example, the classical double fusion hypothesis, which states that both images on one retina are fused with the single image on the other (Hering, 1865, cited in Ono \& Mapp, 1995; Ogle, 1962), cannot account for the magnitude and the direction of the perceived depth re-

This research was supported by Grant-in-Aids for Scientific Research (7610068) provided by the Japanese Ministry of Education, Science, and Culture. K. Shimono worked on this study while he was at the Communications Research Centre, Ottawa, Canada, as a visiting researcher from the Department of Information Engineering and Logistics, Tokyo University of Mercantile Marine. The authors wish to thank Atsuki Higashiyama, two anonymous reviewers, and the editor for their critical review and helpful comments on an earlier version of the manuscript. Correspondence should be addressed to K. Shimono, Department of Information Processing Engineering and Logistics, Tokyo University of Mercantile Marine, Ettchujima 2-1-6, Koto-ku, Tokyo, Japan, 136 (e-mail: shimono@ipc.tosho-u.ac.jp). ported by observers (see, e.g., Gettys \& Harker, 1967; Howard \& Ohmi, 1992; Ono et al., 1992; Ono \& Wade, 1985; cf. Gillam, Blackburn, \& Cook, 1995).

Aside from the double fusion hypothesis, other hypotheses have been examined in the past, but the results have been diverse and inconclusive. For example, Nakayama and Shimojo (1990) examined the hypothesis asserting that the visual system utilizes an occlusion cue in the Wheatstone-Panum limiting case and showed that the magnitude and direction of the perceived depth were consistent with the occlusion hypothesis. However, Howard and Ohmi (1992), as well as Shimono, Nakamizo, and Ida (1994a, 1994b) showed that the occlusion hypothesis cannot account for the magnitude of perceived depth, although it can account for the direction. Howard and $\mathrm{Ohmi}$ provided results that were consistent with another hypothesis that asserts that the perceived depth in the Wheatstone-Panum limiting case is based upon a camouflage cue. Nakayama and Shimojo and Ono et al. (1992), on the other hand, reported data showing that the visual system does not utilize the camouflage cue. Complicating matters, Howard and Ohmi and Howard and Rogers (1995) claimed that misconvergence plays an important role in depth perception in the WheatstonePanum limiting case; however, this vergence-error hypothesis cannot account for the results obtained by Gillam 


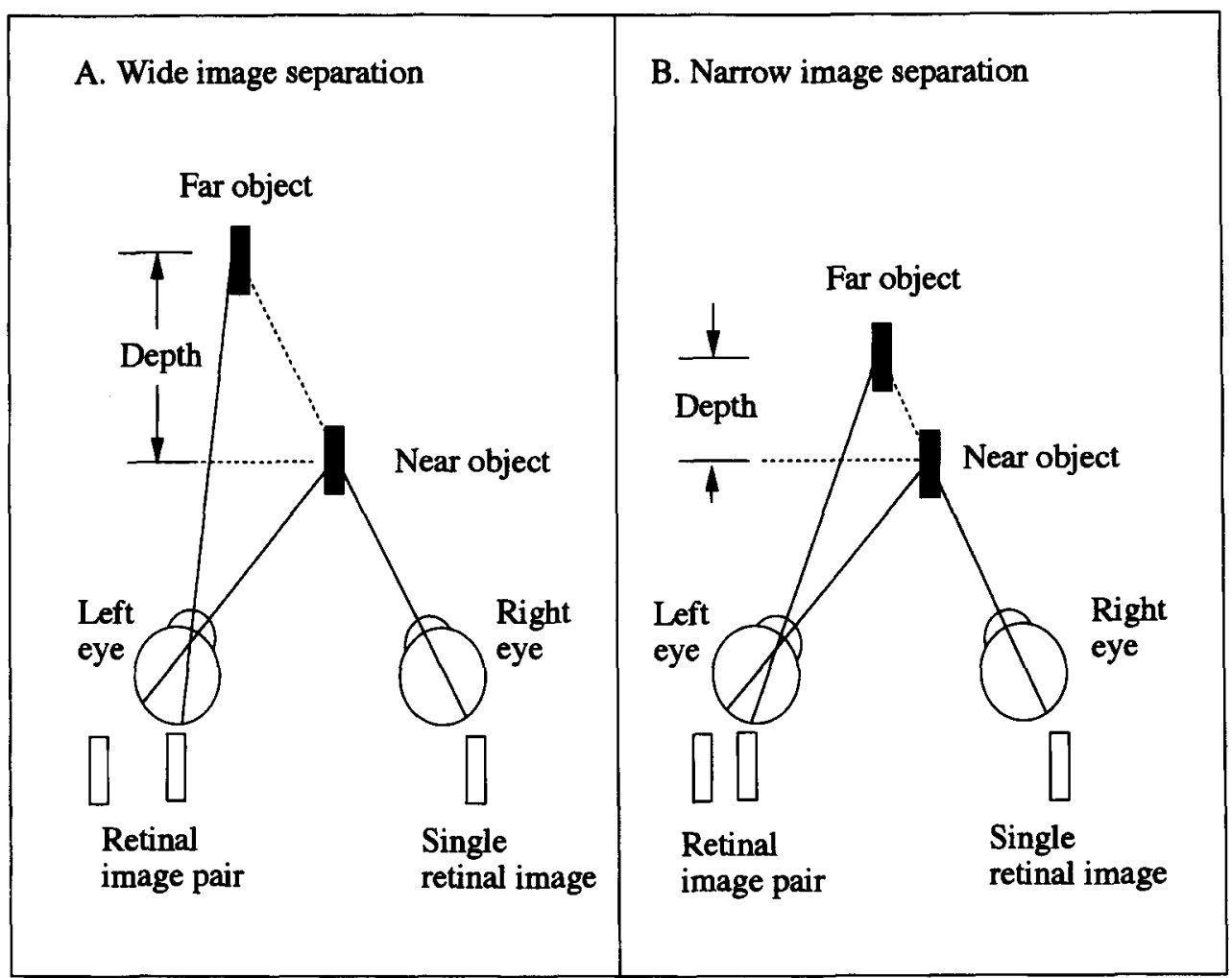

Figure 1. Two stimuli along a visual line of the right eye produce a single image in the right eye and two images in the left eye. As the magnitude of depth between the two stimuli is increased, the separation of the two images in the left eye becomes wider. If the visual system processes information based on this geometrical relationship, according to the occlusion and camouflage hypotheses, the perceived depth would be larger with a wide image separation (A) than with a narrow image separation (B).

et al. (1995). These divergent and sometimes contradictory results suggest that, in the Wheatstone-Panum limiting case, (1) more than one cue is probably responsible for sensations of depth and (2) individuals may differ as to which cue they utilize.

In the present study, we used a relatively large number of observers to examine predictions based on the three hypotheses. Using a large number of observers would minimize effects arising from individual differences and would better delineate the roles of occlusion, camouflage, and vergence. Thus, in Experiment 1, predictions based on the binocular occlusion and camouflage hypotheses were examined using 16 observers. In Experiment 2, a prediction based upon the vergence-error hypothesis was examined using 45 observers.

\section{EXPERIMENT 1}

The occlusion and camouflage hypotheses are based on the idea that the Wheatstone-Panum limiting case represents two objects on the visual line of one eye, as depicted in Figure 1. The figure shows how an image pair is formed in one eye (the left, in this case) and how a single image is formed in the other eye (the right). According to both hypotheses, one of the two images in the left eye fuses with the single image in the right eye; the remaining image in the left eye has no counterpart in the right eye, and is not fused. Thus, of the two perceived objects, one is generated from the fusion of two images, and the other is generated from an image that is not fused. Furthermore, according to both hypotheses, the perceived depth between the objects should covary with the spatial separation between the images of the image pair in the left eye. As illustrated in Figure 1, both hypotheses predict that the magnitude of perceived depth will be larger with a wide image separation (panel A), than with a narrow image separation (panel B).

The occlusion and camouflage hypotheses differ in their explanations of how depth is perceived in the Wheatstone-Panum limiting case. According to the occlusion hypothesis, the image on the temporal side of the image pair in the left eye fuses with the single image in the right eye, as shown in Figure $2 \mathrm{~A}$. It is as if the visual system interpreted the stimulus configuration as a near (fused) object occluding a distant (nonfused) object. On the other hand, according to the camouflage hypothesis, the image on the nasal side of the image pair in the left eye fuses with the single image in the right eye, as shown in Figure 2B. It is as if the visual system interpreted the stimulus configuration as a near (nonfused) object being 


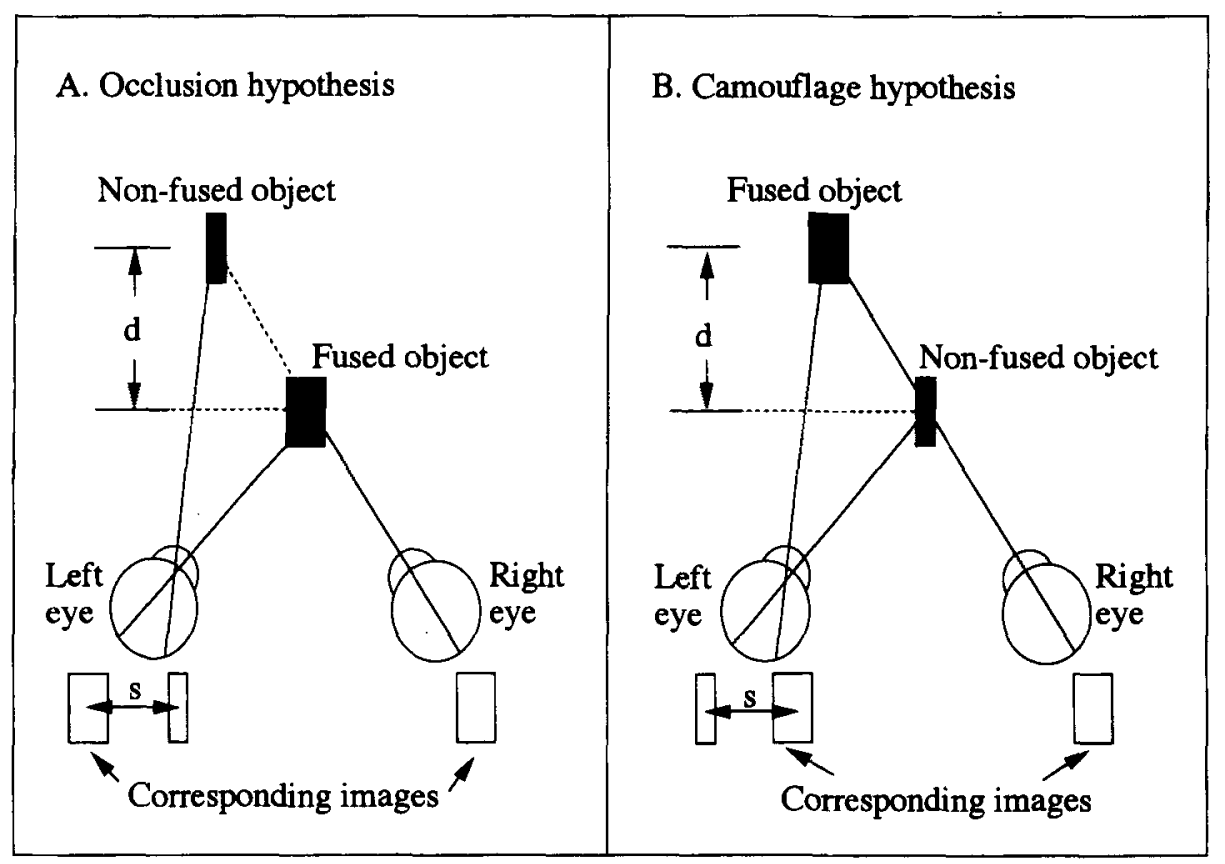

Figure 2. Explanation of how depth is perceived on the basis of the occlusion hypothesis $(A)$ and the camouflage hypothesis (B). According to the occlusion hypothesis, the image on the temporal side of the two images on one retina is assumed to be fused with the single image on the other retina. According to the camouflage hypothesis, it is the image on the nasal side that is fused. The occlusion hypothesis predicts that the nonfused image will appear behind, whereas the camouflage hypothesis predicts that the nonfused image will appear in front of the fused image. Both hypotheses predict that the magnitude of the perceived depth, labeled " $d$ " in the figure, will covary with the separation of the two retinal images, labeled "s" in the figure.

camouflaged by a distant (fused) object. Note that the two hypotheses differ in their predictions of whether the fused object will be perceived in front of or behind the nonfused object; specifically, the occlusion hypothesis predicts that the fused object will appear in front, while the camouflage hypothesis predicts that the fused object will appear behind.

To examine the two hypotheses and predictions, we measured the magnitude and direction of perceived depth in the Wheatstone-Panum limiting case, while manipulating one of the image pair, either on the temporal or the nasal side, so as to fuse with the single image on the other retina. Position of the image pair was manipulated in such a way that either the temporal or the nasal image was brought into binocular correspondence with the single image on the other retina. The stimulus configuration with the temporal image in correspondence will be referred to as the occlusion configuration (Figure 2A), and the stimulus configuration with the nasal image in correspondence will be referred to as the camouflage configuration (Figure 2B). ${ }^{2}$ It was expected that images on corresponding retinal points would fuse and appear as an object in the fixation plane. To further increase the likelihood that the images on corresponding retinal points would fuse, the images were made larger than the noncorresponding image. We expected that by making images on corre- sponding retinal points larger than that at the noncorresponding point, it would be easier for the visual system to interpret the corresponding images as coming from an occluding or camouflaging object (Ono et al., 1992; Ono \& Wade, 1985). Thus, we manipulated both image position and image size to increase the likelihood that occlusion cues would operate in the occlusion configuration and that camouflage cues would operate in the camouflage configuration.

\section{Method}

Stimuli and Apparatus. Stereoscopic stimuli were generated by a NEC PC-9801 computer and were displayed on an NEC color monitor (PC-KD853). The center of the monitor was at eye level, and viewing distance was $100 \mathrm{~cm}$. Polarized filters made the left half of the screen visible only to the right eye, and the right halffield visible only to the left eye. Convergence distance was about $40 \mathrm{~cm}$, with a -1.5 -D lens placed in front of each eye to match accommodation to the convergence distance. In addition, a variable diopter prism was positioned in front of the right eye, allowing image location to be adjusted on the retina.

The stimuli consisted of 36 test stereograms and 14 reference stereograms. The test stereograms contained the Wheatstone-Panum limiting case, with half the stereograms in the occlusion configuration, and the other half in the camouflage configuration. The reference stereograms depicted the general case of two objects visible to both eyes. Estimates of depth obtained with the reference stereograms were used to standardize estimates of perceived depth ob- 


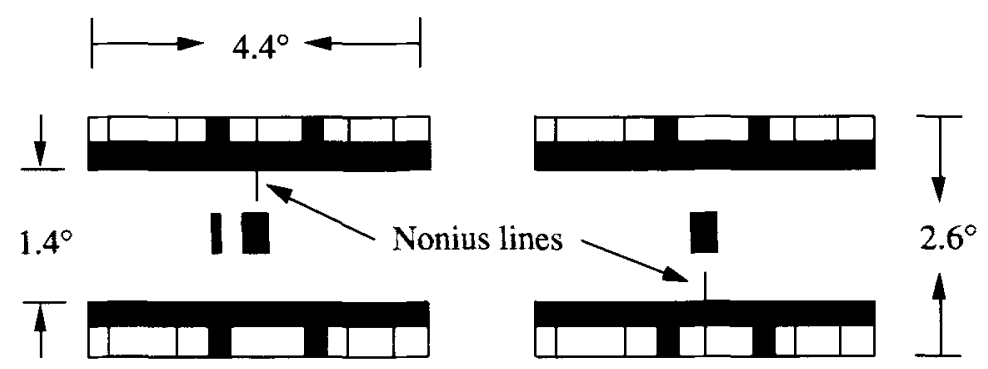

Figure 3. Example of a stereogram used in Experiments 1 and 2 (not drawn to scale).

tained with the test stereograms; a preliminary study showed that there can be large individual differences in estimates of depth based on the test stereograms. Details of the test and reference stereograms are provided below.

In the test stereograms, one half-field contained two vertical bars and the other half-field contained one single bar. The height of the bars was fixed at $20.8^{\prime}$ of arc, and the width of the bars was varied. For the single bar in one half-field as well as for its corresponding bar in the other half-field, the width of the bars was $10.4^{\prime}, 31.2^{\prime}$, or $52.0^{\prime}$ of arc, and for the noncorresponding bar in the other halffield, the width of the bar was fixed at $5.2^{\prime}$ of arc. We will refer to the stereogram containing the bar width of $10.4^{\prime}, 31.2^{\prime}$, or $52.0^{\prime}$ of arc as the narrow, intermediate width, or wide bar test stereogram, respectively. In addition to manipulating the width of the bars, the spatial separation between the two bars in a given half-field was varied at $10.4^{\prime}, 20.8^{\prime}$, and $31.2^{\prime}$ of arc; these magnitudes of separation will be referred to as small, medium, and large, respectively. In general, there was an equal number of test stereograms for the different combinations of stimulus configuration, bar width, and bar separation, with counterbalancing of the pair of bars in the left and right half-fields.

In contrast to the test stereograms, the reference stereograms contained two vertical bars in both half-fields. The height of the bars was fixed at $20.8^{\prime}$ of arc, but the width of the bars was varied, depending on the disparity in the stereogram, which could be zero, crossed $20.8^{\prime}$ of arc, or uncrossed $20.8^{\prime}$ of arc. For a given half-field of the stereograms with zero disparity, the width of one of the bars was $10.4^{\prime}$ of arc and the other bar was $5.2^{\prime}$ of arc. Bars in the other half-field were of corresponding widths. For a given half-field of the stereograms with nonzero disparity, the width of one of the bars was varied at $10.4^{\prime}, 31.2^{\prime}$, and $52.0^{\prime}$ of arc, while the other bar was fixed at $5.2^{\prime}$ of arc. Bars in the other half-field were of corresponding widths. In the stereograms with nonzero disparity, the wider bar was set in the center of each half-field, and the narrower bar was positioned left or right of the center position. We will refer to the stereograms containing bar widths of $10.4^{\prime}, 31.2^{\prime}$, and $52.0^{\prime}$ of arc as the narrow, intermediate width, and wide bar reference stereograms, respectively.

Aside from the vertical bars of interest, both half-fields of each test and reference stereogram were bounded on the top and bottom by a bandlike pattern, as shown in Figure 3 . The bands facilitated binocular fusion and helped "lock" convergence. A Nonius line $\left(3.9^{\prime} \times 54.6^{\prime}\right.$ of arc) was placed at the center of the top band in one half-field, and at the center of the bottom band in the other halffield. The Nonius lines were used to monitor eye position. When the lines were collinear, it was assumed that convergence was at the desired position. (For discussions of the Nonius method, see Ogle, Martens, \& Dyer, 1967; Shimono, Ono, Saida, \& Mapp, 1998.)

Procedure. Observers were asked on each trial (1) to report whether or not the two bars appeared in the same frontoparallel plane and, if they did not, which bar appeared closer, and (2) to report the magnitude of depth between the two bars in millimeters or in centimeters. ${ }^{3}$ (Observers sometimes reported seeing double images of the wide bar for the reference stereogram. That is, they saw three bars. These observers were instructed to report the perceived depth between the single narrow bar and the two wide bars.) $\mathrm{Ob}$ servers were instructed to respond only when the Nonius lines were aligned. If observers had difficulty adjusting their convergence to make the Nonius lines collinear, the variable diopter prism in front of the right eye was adjusted until the lines appeared collinear.

Observers were given two or three blocks of trials as practice. Each block consisted of 14 reference stereograms presented in a random order. During practice, observers were given feedback as to the correct direction of depth. Observers who responded correctly, for all 14 stereograms in the last block of trials, were allowed to proceed in the study.

The experiment consisted of two sessions - one with 14 reference stereograms and the other with 36 test stereograms. The presentation order of the reference and test sessions was pseudorandomized. Within each session, the stereograms were presented in a random order, with one repetition of each stereogram. During these sessions, feedback as to the correct direction of depth was not given to observers. For each trial, the stereograms were presented for as long as observers needed to align the Nonius lines and respond with confidence. The observers were allowed to take a rest any time during the two sessions. The experiment took about an hour to complete.

Observers. Twenty-two university students enrolled in a general psychology class volunteered for the experiment. After screening for correct responses as to the direction of depth in the reference stereograms, 16 students were allowed to continue in this experiment. The 4 females and 12 males ranged in age from 18 to 23 years, and they reported having normal or corrected-to-normal visual acuity and normal binocular stereopsis.

\section{Results and Discussion}

The basic unit for analysis was the average of the normalized depth values, for each experimental condition and observer. Normalization proceeded as follows. Reported depth values obtained with the test stereograms were assigned a positive or a negative sign, depending on whether the nonfused bar was perceived to be behind or in front of the fused bar, respectively. Then, the signed values were divided by the reported depth values obtained with the reference stereograms. Specifically, positively signed values were divided by the reported depth values for reference stereograms with uncrossed disparities, and negatively signed values were divided by the reported depth values for reference stereograms with crossed disparities.

We performed a three-way analysis of variance (ANOVA), with repeated measures on the stimulus con- 


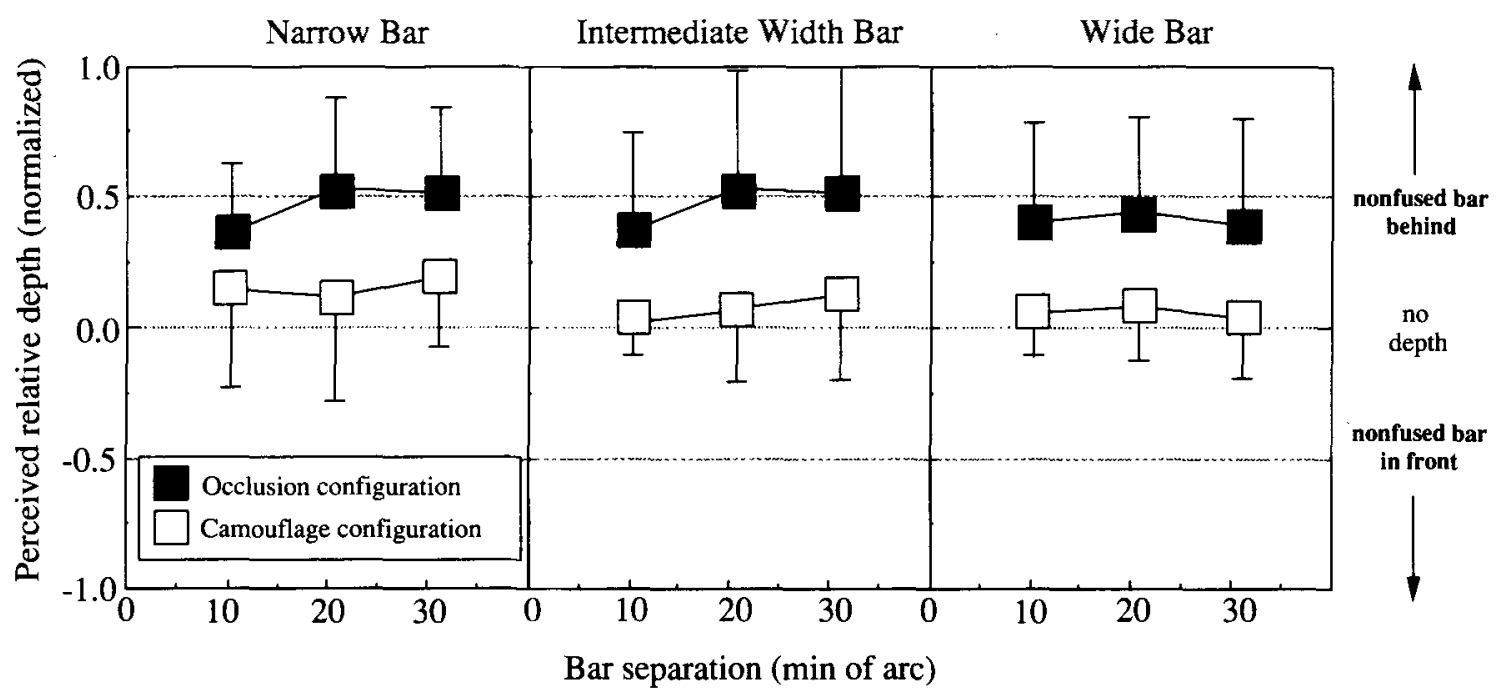

\begin{abstract}
Figure 4. The normalized depth values of the nonfused stimulus relative to the fused stimulus as a function of the horizontal separation between two bars in one field of a test stereogram. Separate graphs are shown for the narrow, intermediate width, and wide bar conditions. Each symbol represents the mean value across 16 observers. The solid and open squares indicate that the data are from the occlusion and camouflage configurations, respectively. The vertical lines indicate the standard deviations.
\end{abstract}

figurations, the separations of the two bars in one halffield, and the bar widths, on the averages of the normalized values. The analysis showed that the main effect of stimulus configuration was statistically significant $[F(1,15)=34.02, p<.001]$, whereas the other two main effects as well as all their interactions were not statistically significant.

The significant main effect of stimulus configuration can be seen in Figure 4, which shows the mean normalized value over 16 observers as a function of the separation of the two bars in one field, for each of the three bar-width conditions. The results indicate that the nonfused bar was seen behind the fused bar in the occlusion configuration and it was seen near the fused bar in the camouflage configuration. The normalized values were relatively constant in each of the three bar-width conditions, with a mean normalized value of .45 for the occlusion configuration and a mean normalized value of .09 for the camouflage configuration. $T$ tests showed that the magnitudes of perceived depth were significantly greater than zero for all occlusion configurations [narrow bar condition- $t(15)=$ $5.587, p<.001, t(15)=5.813, p<.001, t(15)=6.191$, $p<.001$; intermediate bar width condition $-t(15)=$ $4.489, p<.001, t(15)=4.716, p<.001, t(15)=3.987$, $p<.01$; wide bar condition- $t(15)=4.355, p<.001$, $t(15)=4.497, p<.001, t(15)=3.775, p<.01$, for the small, medium, and large bar separation conditions, respectively] as well as in one camouflage configuration in which the bar width was "narrow" and the bar separation was "large" $[t(15)=2.806, p<.05]$.

Are the results from the occlusion configuration consistent with predictions based on the occlusion hypothesis? The present results indicate that the direction of perceived depth is consistent with the prediction, but the magni- tude of perceived depth is not. In the occlusion configuration, the nonfused bar appeared behind the fused bar, as predicted, but the perceived depth between them did not change with the three different bar separations used in this study (see Figure 4). These results suggest that the visual system utilizes binocular occlusion as a depth cue and that this occlusion cue provides depth-order information but not depth-magnitude information.

The present results with respect to depth magnitude are apparently at odds with those reported by Nakayama and Shimojo (1990); they found that the occlusion cue provided depth-magnitude information in addition to depth-order information. In their study, only 3 observers were used. In contrast, 16 observers were used in the present study. The apparently different results between the two studies may be due to individual differences. It is also possible that the difference in results is due to the experimental procedure they used. In their procedure, observers were asked to adjust the disparate ("fused") stimulus to the same depth plane as the nonfused stimulus while fixating the Nonius stimulus. With this procedure, observers may have changed their convergence, because it is difficult to do the adjustment while keeping binocular eye position in one fixation plane. This possibility is supported by the fact that observers in their study did not report seeing double images even when "disparity" was near $30^{\prime}$ of arc. This value is about three times larger than the estimate of Panum's fusional area for a simple narrow stimulus (Mitchell, 1966; Nakamizo, Shimono, Kondo, \& Ono, 1994). Thus, it is plausible that eye position may not have been where it was supposed to be. If this is the case, the results of Nakayama and Shimojo may be due to eye position rather than use of the occlusion cue; eye position can be an effective cue for depth magnitude (see, e.g., 


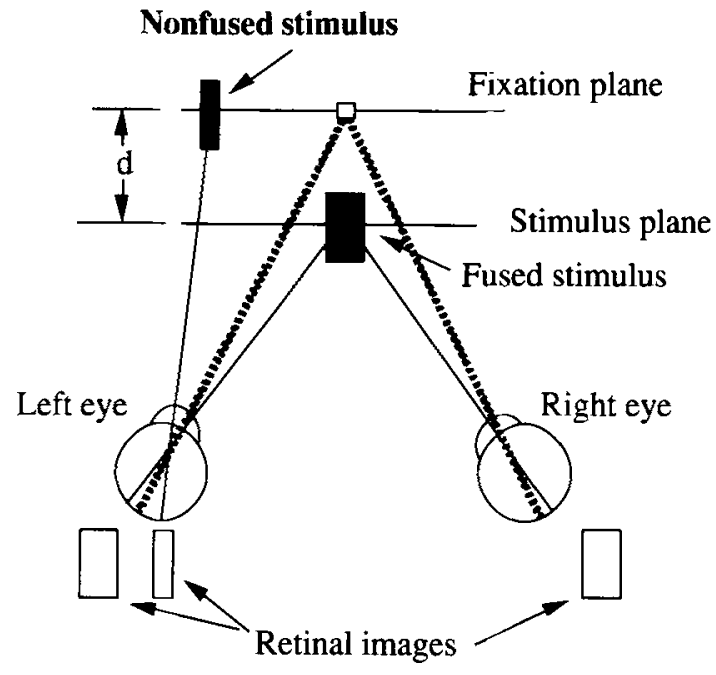

Figure 5. Explanation of how depth is perceived on the basis of the vergence-error hypothesis. The hypothesis asserts that (1) one of the two images on one retina fuses with the single image on the other retina, with the remaining image having no counterpart to fuse, and (2) the fused stimulus is located in the stimulus plane and the nonfused stimulus is located in the fixation plane. According to the hypothesis, the visual system utilizes the disparity between the fused stimulus and the actual fixation point in such a way that the depth, labeled " $d$ " in the figure, between the two perceived stimuli will covary with the magnitude of the vergenceinduced disparity.

Howard \& Rogers, 1995; Kaufman, 1976; Shimono, 1993). We examined the effect of eye position on perceived depth in the Wheatstone-Panum limiting case in Experiment 2.

Are the results from the camouflage configuration consistent with predictions based on the camouflage hypothesis? The results indicate that the nonfused bar appeared near the frontoparallel plane in which the fused bar was located, and that the depth between the two bars did not change with the three different bar separations that were used in this study (see Figure 4). The results are not consistent with predictions based on the camouflage hypothesis and fail to confirm the results of Howard and Ohmi (1992), who found that binocular camouflage cues provide depth-order information. One interpretation of the present results is that manipulation of the size of the corresponding images was not effective in making the camouflage cue explicit. However, our results are consistent with those of Nakayama and Shimojo (1990), Ono et al. (1992), and Shimono et al. (1994a, 1994b). Considered together, the results lead us to conclude that binocular camouflage, if at all, is a very weak cue in the WheatstonePanum limiting case.

\section{EXPERIMENT 2}

In this experiment, we examined the vergence-error hypothesis, which states that depth perceived in the
Wheatstone-Panum limiting case is a result of misconvergence. According to this hypothesis, (1) one of the two images on one retina fuses with the single image on the other retina, and the remaining image, without a counterpart, is not fused; (2) there is misconvergence of the fused stimulus; (3) the nonfused stimulus appears in the frontoparallel plane where the eyes are converged; and (4) the fused stimulus appears in depth relative to the fixation plane, as a result of the disparity produced by misconvergence. Figure 5 depicts the case in which there is overconvergence and the fused stimulus appears in front of the nonfused stimulus. Not depicted in the figure is the case of underconvergence, in which the fused stimulus appears behind the nonfused stimulus. The magnitude of the perceived depth between the fused stimulus and the nonfused stimulus would be determined by the extent of the vergence error - that is, by the vergence-induced disparity between the fused stimulus and the "actual" fixation point. (For a more detailed explanation of the hypothesis, see Howard \& Rogers, 1995, pp. 519-520; Kaufman, 1976).

In the present experiment, we manipulated the magnitude of the vergence-induced disparity and measured the perceived depth in the Wheatstone-Panum limiting case. Vergence-induced disparity was manipulated by systematically varying the location of the fixation plane. Furthermore, we made either the temporal or the nasal image of the image pair on one retina the same size as the single image on the other retina, expecting that images of the same size would fuse more readily. (We will refer to the stimulus configurations as the occlusion and the camouflage configurations, respectively.)

\section{Method}

Stimuli and Apparatus. The apparatus was the same as that used in-Experiment 1. The stimuli consisted of 252 test stereograms and 42 reference stereograms. The test stereograms contained the Wheatstone-Panum limiting case with half the stereograms in the occlusion configuration and the other half in the camouflage configuration. As in Experiment 1, estimates of depth obtained with the reference stereograms were used to standardize reports of perceived depth obtained with the test stereograms.

The test stereograms were the same as those in Experiment 1 except that the two wider bars, which are assumed to be fused by observers, had horizontal disparity with respect to the Nonius lines. When the Nonius lines were aligned, it was assumed that disparity was induced between the actual fixation point and the "fused" bar. There were seven vergence-induced disparities and three bar widths: narrow, intermediate width, and wide, corresponding to $10.4^{\prime}, 31.2^{\prime}$, and $52.0^{\prime}$ of arc, respectively. The vergence-induced disparities for the narrow bar test stereograms were $0^{\prime}, 5.2^{\prime}, 7.8^{\prime}$, and $10.4^{\prime}$ of arc crossed and $5.2^{\prime}, 7.8^{\prime}$, and $10.4^{\prime}$ of arc uncrossed. Disparities for the intermediate and wide bar test stereograms were $0^{\prime}, 5.2^{\prime}, 10.4^{\prime}$, and $15.6^{\prime}$ of arc crossed and $5.2^{\prime}, 10.4^{\prime}$, and $15.6^{\prime}$ of arc uncrossed. (The disparity range used in the narrow bar test stereograms was made smaller than those in the other two test stereograms because in a preliminary study, observers reported double vision when the disparity was $15.6^{\prime}$ of arc.) For each test stereogram, there were also three bar separations: small, medium, and large, corresponding to $10.4^{\prime}, 20.8^{\prime}$, and $31.2^{\prime}$ of arc, respectively. Thus, there was an equal number of test stereograms for the differ- 
Table 1

Main Effects and Interactions of the Normalized Depth Values for Experiment 2

\begin{tabular}{|c|c|c|c|c|c|c|c|c|c|}
\hline \multirow[b]{3}{*}{ Effect } & \multicolumn{9}{|c|}{ Bar Width Conditions } \\
\hline & \multicolumn{3}{|c|}{ Narrow Bar } & \multicolumn{3}{|c|}{ Intermediate Width Bar } & \multicolumn{3}{|c|}{ Wide Bar } \\
\hline & $d f$ & $F$ & $p$ & $d f$ & $F$ & $\underline{p}$ & $d f$ & $F$ & $p$ \\
\hline Vergence-induced disparity (VD) & 6,84 & 8.001 & .000 & 6,84 & 15.850 & .000 & 6,84 & 16.214 & .000 \\
\hline Stimulus configuration (SC) & 1,14 & 20.520 & .001 & 1,14 & 11.074 & .005 & 1,14 & 21.360 & .000 \\
\hline Bar separation (BS) & 2,28 & .280 & .758 & 2,28 & .983 & .387 & 2,28 & 1.884 & .171 \\
\hline $\mathrm{VD} \times \mathrm{SC}$ & 6,84 & 3.261 & .006 & 6,84 & 3.306 & .006 & 6,84 & 1.320 & .258 \\
\hline $\mathrm{VD} \times \mathrm{BS}$ & 12,168 & 1.850 & .044 & 12,168 & 2.274 & .011 & 12,168 & 4.381 & .000 \\
\hline $\mathrm{SC} \times \mathrm{BS}$ & 2,28 & 1.276 & .294 & 2,28 & 2.174 & .132 & 2,28 & 5.850 & .008 \\
\hline $\mathrm{VD} \times \mathrm{SC} \times \mathrm{BS}$ & 12,168 & 1.386 & .177 & 12,168 & 2.195 & .014 & 12,168 & .550 & .879 \\
\hline
\end{tabular}

ent combinations of vergence-induced disparity, stimulus configuration, bar width, and bar separation, with counterbalancing of the pair of bars in the left and right half-fields.

As with the test stereograms, three bar widths were used in the reference stereograms; narrow (10.4' of arc), intermediate width (31.2' of arc), and wide (52.0' of arc). The height of the vertical bars in the stereogram was $20.8^{\prime}$ of arc, the same as for the test stereograms. The disparities for the narrow bar reference stereograms were $0^{\prime}, 10.4^{\prime}, 15.6^{\prime}$, and $20.8^{\prime}$ of arc crossed and 10.4', 15.6', and $20.8^{\prime}$ of arc uncrossed. The disparities for the intermediate and wide bar reference stereograms were $0^{\prime}, 10.4^{\prime}, 20.8^{\prime}$, and $31.6^{\prime}$ of arc crossed and $10.4^{\prime}, 20.8^{\prime}$, and $31.6^{\prime}$ of arc uncrossed. (The disparity range used in the narrow bar reference stereogram was different from those in the other two reference stereograms, because in a preliminary study observers reported difficulties in seeing depth when the disparity was $31.6^{\prime}$ of arc.) In each stereogram, the wider bar was set in the center of each half-field, and the narrower bar was positioned left or right of the center position, as in Experiment 1 .

Procedure. Observers were asked on each trial (1) to report whether or not the two perceived bars appeared in the same plane, and if they did not, which bar appeared closer, and (2) to report the magnitude of depth between the two bars in millimeters or in centimeters. When observers reported seeing three bars, they were instructed to report the perceived depth between the single narrow bar and the two wide bars. Observers were instructed to respond only when the Nonius lines were seen aligned. If they had difficulty adjusting their convergence to make the Nonius lines collinear, the variable diopter prism in front of the right eye was adjusted until the lines appeared collinear.

Observers were given two or three blocks of trials as practice. Each block consisted of 14 narrow, intermediate width, or wide bar reference stereograms. During practice, observers were given feedback as to the correct direction of depth. Observers who responded correctly, for all 14 stereograms in the last block of trials, were allowed to proceed in this experiment.

The experiment consisted of three sessions--one with the narrow bar reference and test stereograms, a second with the intermediate width bar reference and test stereograms, and a third with the wide bar reference and test stereograms. Each session consisted of the presentation of 14 reference stereograms and 84 test stereograms. The test stereograms were presented in a random order and so were the reference stereograms. The order of presentation of the test and reference stereograms was counterbalanced. For each trial, the stereograms were presented for as long as observers needed to align the Nonius lines and respond with confidence. The observers were allowed to take a rest at any time during the session, which took about an hour to complete. As in Experiment 1, feedback as to the correct direction of depth was not given to observers during the actual test sessions.

Observers. Fifty-two university students enrolled in a general psychology class volunteered for the experiment. After screening for correct responses as to the direction of depth with the reference stereograms, 45 students were allowed to continue in this experiment. Fifteen observers were assigned to each of the three sessions. The 5 females and 40 males who were tested ranged in age from 18 to 40 years and reported having normal or corrected-to-normal acuity and stereopsis.

\section{Results and Discussion}

The reported depth values from each observer were normalized as described in Experiment 1, except that the values were divided by reference values obtained using the corresponding reference stereogram with $10.4^{\prime}$ of arc disparity for each of the three bar-width conditions. The normalized values were averaged for each experimental condition and observer. The average of the normalized values was the basic unit of analysis.

We performed a three-way ANOVA on the averages of the normalized values, with repeated measures on the vergence-induced disparity, the stimulus configuration, and the separation of the two bars in one half-field. The analysis was done separately for each of the three barwidth conditions. Results of the analysis are shown in Table 1. In each of the three conditions, the main effects of vergence-induced disparity and stimulus configurations were statistically significant, while the main effect of bar separation was not.

The significant main effects can be seen in Figure 6, which shows the mean normalized values averaged over the 15 observers as a function of vergence-induced disparity. The left and right panels show results for the occlusion and camouflage configurations, respectively, and the upper, middle, and lower panels show results for the narrow, intermediate width, and wide bar conditions, respectively. As shown in each panel, the mean normalized values covaried with the vergence-induced disparity as a whole, and they were larger in the occlusion configuration than in the camouflage configuration at all vergenceinduced disparity conditions.

However, the significant main effects are qualified by the two-way and three-way significant interactions (see Table 1). Most of the significant interactions can also be seen in Figure 6. The two-way interaction between vergence-induced disparity and bar separation in each of the three bar separation conditions can be seen in its respective panel of Figure 6: The normalized values decreased 
Narrow Bar

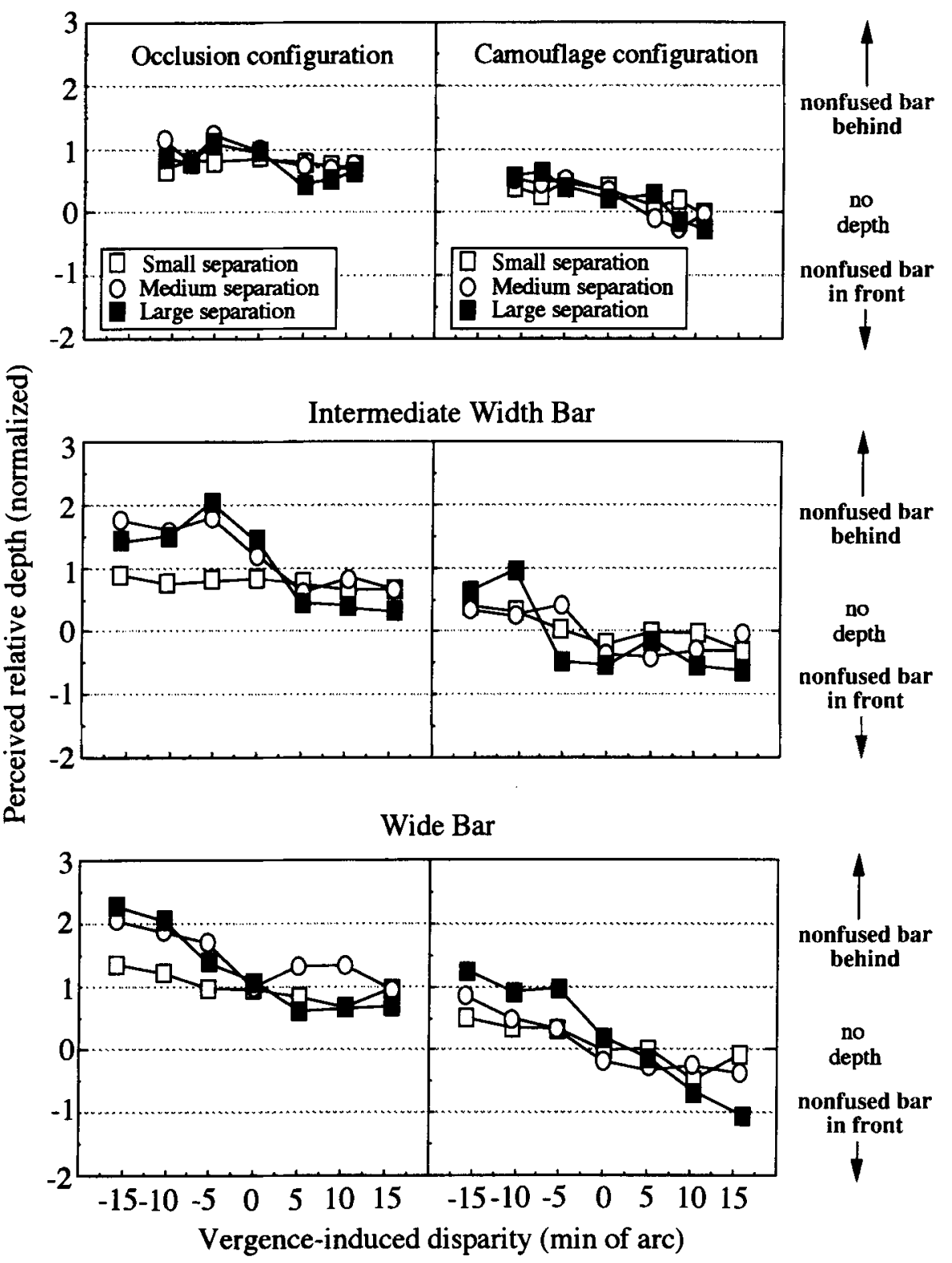

Figure 6. The normalized depth values of the nonfused stimulus relative to the fused stimulus as a function of the vergence-induced disparity for the narrow, intermediate width, and wide bar conditions. The left and right panels show the results for the occlusion and camouflage configurations, respectively. Each symbol represents the mean value across 15 observers. The open squares, open circles, and solid squares indicate the means for small (10.4' of arc), medium (20.8 of arc), and large (31.2' of arc) bar separation conditions, respectively. In the figure, the standard deviations of each point are not shown. The means of the 21 SDs for data in the upper, middle, and lower left panels are $0.66,0.95$, and 0.94 , respectively, and those for data in the upper, middle, and lower right panels are $0.61,1.57$, and 1.31 , respectively.

differently among the three bar separation conditions and this is most evident in the lower panels. The two-way interaction between vergence-induced disparity and stimulus configuration in the narrow bar condition can be seen in the top panels: The normalized values decreased more rapidly in the camouflage configuration than in the occlusion configuration as a function of the vergence- induced disparity. The three-way interaction in the intermediate width bar condition can be seen in the middle panels: The normalized values remained relatively constant across vergence-induced disparities only when the bar separation was "small" in the occlusion configuration. Although the two-way interaction between stimulus configuration and bar separation in the wide bar condition 


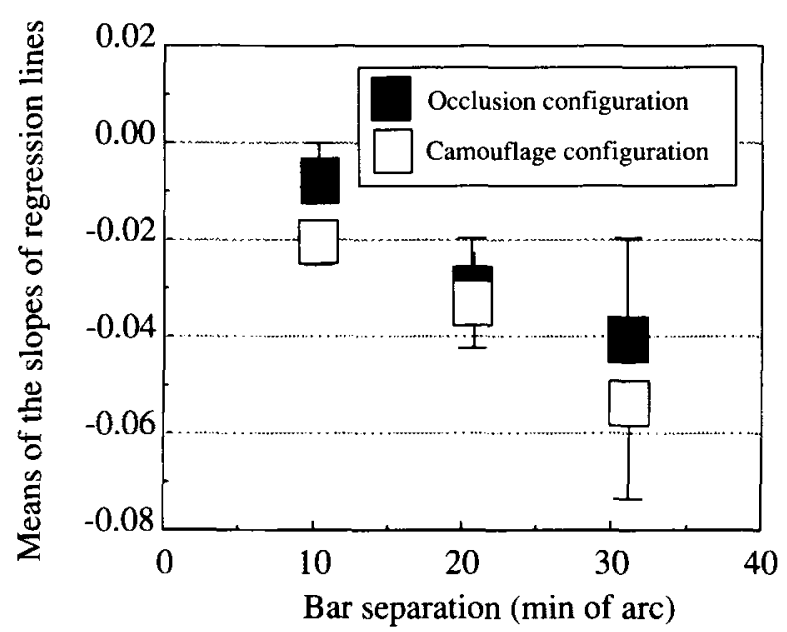

Figure 7. The means of the slopes of the regression lines as a function of the separation between the two bars in one field of a test stereogram. Each symbol represents the mean slope over the three bar-width conditions for the occlusion (open squares) and camouflage (filled squares) configurations, respectively. The vertical lines indicate the standard deviations.

cannot be seen in the lower panels, an analysis of simple main effects revealed that the normalized depths in the three bar separation conditions were significantly different in the occlusion configuration $[F(2,28)=5.131, p<$ $.05]$ and not in the camouflage configuration $(F<1)$.

For further analysis, we calculated the slopes of the regression lines for the data shown in Figure 6, except that the calculations were done for each observer using the averaged normalized values. A three-way ANOVA on the slopes of the regression lines was performed, with stimulus configuration and bar separation as the repeated measure and with bar width as the between-subjects measure. It was found that main effects of stimulus configuration and bar separation were statistically significant $[F(2,84)=21.45, p<.001$, and $F(1,42)=4.492, p<.05$, respectively], whereas the main effect of bar width and the interactions were not significant. The main effects of stimulus configuration and bar separation can be observed in Figure 7, which depicts the mean slopes of regression lines, averaged across the three bar-width conditions, as a function of the separation of the two bars. Note that symbols for the occlusion configuration (filled squares) are above those of the camouflage configuration (open squares), and that the absolute magnitudes of the slopes increase with increasing bar separation. The difference in slope between any two bar separation conditions was statistically significant for all comparisons (Tukey HSD tests, $p<.05$ ).

The obtained covariation between the normalized depth value and the vergence-induced disparity, as graphically depicted in Figure 6, is consistent with the prediction based on the vergence-error hypothesis ( $\mathrm{Lau}$, cited in Howard \& Rogers, 1995; Howard \& Rogers, 1995; Kaufman, 1976; Shimono, 1993). The covariation suggests that vergence-induced disparity, like binocular disparity, pro- vides both depth-magnitude and depth-order information. However, note that the vergence-induced disparity is not a "strong" depth cue relative to binocular disparity. This can be demonstrated by comparing the slopes of the regression lines calculated for the test stereograms with those for the reference stereograms, on the assumption that the latter slopes reflect the effect of binocular disparity. In calculating the slopes for the reference stereograms, we normalized the reported values with the values obtained for the smallest disparity (10.4' of arc), taking into account the disparity type (crossed or uncrossed). We computed the slopes of the regression lines using data from the seven disparity conditions ( 3 crossed, 0 , and 3 uncrossed disparities) for each of the three reference stereograms. The calculated slopes for the narrow, intermediate width, and wide bar reference stereograms were $-0.103,-0.110$, and -0.104 , respectively. The mean value $(-0.105)$ of these three slopes was three times larger than the mean value $(-0.031)$ of the six experimental conditions shown in Figure 7 . This difference suggests that the vergence-induced disparity is a weaker depth cue than binocular disparity. Given that the vergence-induced disparity is a weak cue, other factors such as stimulus configuration and bar separation will be more likely to play a role in determining perceived depth. The results of the present experiment are consistent with this expectation.

The fact that normalized values in the occlusion configuration were larger than those in the camouflage configuration, as graphically shown in Figure 6, indicates that the stimulus configuration had an effect on perceived depth. Furthermore, the effect of the stimulus configuration differed slightly between the occlusion and camouflage configurations, as shown in Figure 7. Notice that the normalized values changed more rapidly as a function of bar separation in the camouflage configuration than in the occlusion configuration. This result suggests that it is easier for the visual system to utilize the vergenceinduced disparity cue in the camouflage configuration than in the occlusion configuration. This may be the case because there is a potential cue conflict in the occlusion configuration but not in the camouflage configuration. In the occlusion configuration, both the binocular occlusion cue as well as the vergence-induced disparity cue are available. If the vergence-induced disparity cue indicates that the nonfused stimulus lies in front of the fused stimulus, whereas the occlusion cue indicates the opposite, the perceived depth between the two stimuli is likely to be less than when only the vergence-induced disparity cue is available. The difference between the slopes of the occlusion and camouflage configurations shown in Figure 7 is consistent with this interpretation.

Further, the finding that the separation of the two bars in one half-field had an effect on perceived depth in the Wheatstone-Panum limiting case also suggests that the vergence-induced disparity is a more effective cue when the nonfused bar is presented at the peripheral retinal location than when it is presented at the central retinal lo- 
cation. In this study, the nonfused bar was presented at a more peripheral retinal location as the separation of the two bars in one field of a test stereogram was increased. For a given magnitude of vergence-induced disparity, the magnitude of the perceived depth between the fused and the nonfused stimuli increased as the separation of the two bars was increased. However, when the vergenceinduced disparity was zero, the retinal location had no effect on perceived depth. A one-way ANOVA showed that the normalized depths were not statistically significantly different among the three bar separations in all experimental conditions in which the vergence-induced disparity was zero. Thus, the results suggest that perceived depth in the Wheatstone-Panum limiting case depends on the retinal location of the nonfused stimulus when vergence-induced disparity is present.

\section{GENERAL DISCUSSION}

The results of the present study show that stimulus configuration, vergence-induced disparity, and the magnitude of separation between two images on one retina are factors that can contribute to perceived depth in the Wheatstone-Panum limiting case. Experiments 1 and 2 showed that perceived depth depends on whether the temporal or nasal side of the two images is presented so as to fuse with the single image on the other retina. Experiment 2 showed that perceived depth covaries with the vergence-induced disparity. Furthermore, Experiment 2 showed that the separation between the two images can influence perceived depth when vergence-induced disparity is present. The fact that perceived depth in WheatstonePanum limiting case can be influenced by more than one factor suggests that the visual system may be utilizing several depth cues to "see" depth in the WheatstonePanum limiting case.

Although the present results demonstrate that vergence error can play a role in the depth perceived in the Wheatstone-Panum limiting case, the results were obtained using a fixation stimulus to induce the error. When such a fixation stimulus is not present, how might an error in vergence be induced? One possible explanation of how vergence error might be induced in the Wheatstone-Panum limiting case was proposed by Kaufman (cited in Arditi, 1986). Kaufman suggested that "the presence of an adjacent element will tend to cause slight over- or underconvergence due to the eyes' attempt to bring both elements into foveal registration" (Arditi, 1986, pp. 23.21-23.22). Because of this tendency, the eye presented with the two elements will turn and fixation will be directed to an intermediate position between the two elements, leading to misconvergence. According to the vergence-error hypothesis, the misconvergence will produce relative depth between the two perceived elements. This explanation is consistent with reports in the literature that the perceived depth covaries with the separation of the two elements in one eye when eye position is not controlled (e.g., Gettys \& Harker, 1967).

Another interesting finding in this study is that the binocular occlusion cue provides depth-order information but not depth-magnitude information. This finding is interesting because it suggests that the localization of the nonfused area "occurs after stereopsis" for a randomdot stereogram (Julesz, 1971, p. 260). Typically, of two perceived planes in a random-dot stereogram, the nonfused area appears in the depth plane that is farther away (see, e.g., Julesz, 1971). In other words, the nonfused area appears to be localized in the plane with the greater depth as if it has been "captured" by that plane. If it is the case that the nonfused area provides only depth-order information, as our results suggest, this area might be expected to be localized in a fixed depth plane irrespective of the disparity of the depth planes depicted in the stereogram. However, this is not the case; the nonfused area always appears at the depth plane that is farther away, irrespective of its distance from the observer. To explain this localization, we may have to assume a process that locates the nonfused area in the same plane as the farther one. This process must occur after the visual system has processed the disparity of the stereogram and "calculated" the depth between the two perceived depth planes (see Shimojo \& Nakayama, 1990).

Finally, the present results may have theoretical implications. It is usually assumed that small changes in vergence eye position do not play a role in perceived depth between two stimuli with relative disparity, because vergence does not change the magnitude of the relative disparity (Collewijn \& Erkelens, 1990; Howard \& Rogers, 1995). This assumption may be valid in the case of small relative disparity. However, it is not known whether this assumption still holds when the relative disparity is large and double images are perceived. If the double images are treated by the visual system as though they were nonfused stimuli in the Wheatstone-Panum limiting case, the perceived depth between the fused stimulus and the two nonfused stimuli may depend on the magnitude of the vergence-induced disparity (Lau, cited in Howard \& Rogers, 1995; Kaufman, 1976). If this is the case, "stereopsis" with large relative disparity may arise from vergence-induced disparity. This view is consistent with several findings (Krol \& van de Grind, 1986; O'Shea \& Blake, 1987; Tam \& Ono, 1987). O'Shea and Blake found that the direction of the perceived depth observed in random-dot stereograms with large disparities depends on the direction of misconvergence. In addition, Krol and van de Grind (1986) and Tam and Ono (1987) reported that when there is no misconvergence, there is zero perceived depth with line stereograms containing large disparities or "binocular depth mixturè" stimuli (Foley, 1976). These studies suggest that the misconvergence plays an important role in stereopsis with large disparity. 


\section{REFERENCES}

ARDITI, A. (1986). Binocular vision. In K. R. Boff, L. Kaufman, \& J. P. Thomas (Eds.), Handbook of perception and human performance: Vol. 1. Sensory processes and perception (pp. 23.1-23.41). New York: Wiley.

Collewin, H., \& ERKElens, C. (1990). Binocular eye movements and the perception of depth. In E. Kowler (Ed.), Reviews of oculomotor research: Vol. 4. Eye movements and their role in visual and cognitive processes (pp. 213-261). Amsterdam: Elsevier.

Foley, J. M. (1976). Binocular depth mixture. Vision Research, 16, 1263-1267.

Foley, J. M., \& RichaRds, W. (1972). Effects of voluntary eye movement and convergence on the binocular appreciation of depth. Perception \& Psychophysics, 11, 423-427.

GetTYS, C. F., \& HARKER, G, S. (1967). Some observations and measurements of the Panum phenomenon. Perception \& Psychophysics, 2, 387-395

Gillam, B., Blackburn, S., \& Cook, M. (1995). Panum's limiting case: Double fusion, convergence error, or "da Vinci stereopsis." Perception, 24, 333-346.

HowARD, I. P., \& OHMI, M. (1992). A new interpretation of the role of dichoptic occlusion in stereopsis [Arvo Abstracts]. Investigative Ophthalmology \& Visual Science, 33, 1370.

HowARD, I. P., \& Rogers, B. (1995). Binocular vision and stereopsis. New York: Oxford University Press.

Julesz, B. (1971). Foundations of cyclopean perception. Chicago: University of Chicago Press.

Kaufman, L. (1976). On stereopsis with double images. Psychologia, 19, 224-233.

Krol, J. D. (1982). Perceptual ghost in stereopsis: A ghostly problem in binocular vision. Amsterdam: Rodopi.

KROL, J. D., \& VAN DE GRIND, W. A. (1986). Binocular depth mixture: An artefact of eye vergence? Vision Research, 26, 1289-1298.

MrTCHELL, D. E. (1966), A review of the concept of "Panum's fusional areas." American Journal of Optometry \& Archives of the American Academy of Optometry, 43, 387-401.

NaKamizo, S., Shimono, K., Kondo, M., \& Ono, H. (1994). Visual directions of two stimuli in Panum's limiting case. Perception, 23, 1037-1048.

NaKayama, K., \& Shimojo, S. (1990). Da Vinci stereopsis: Depth and subjective occluding contours from unpaired image points. Vision Research, 30, 1811-1825.

OGLE, K. N. (1962). The optical space sense. In H. Davson (Ed.), The eye (Vol. 4, pp. 374-375). New York: Academic Press.

Ogle, K. N., Martens, T. G., \& DyeR, J. A. (1967). Oculomotor imbalance in binocular vision and fixation disparity. Philadelphia: Lea \& Febiger.

ONo, H., \& MAPP, A. P. (1995). A restatement and modification of Wells-Hering's law of visual direction. Perception, 24, 237-252.

ONo, H., Shimono, K., \& ShibuTa, K. (1992). Occlusion as a depth cue in the Wheatstone-Panum limiting case. Perception \& Psychophysics, 51, 3-13.

ONo, H., \& WADE, N. (1985). Resolving discrepant results of the Wheatstone experiment. Psychological Research, 47, 135-142.

O'SHEA, R. P., \& BLAKE, R. (1987). Depth without disparity in randomdot stereograms. Perception \& Psychophysics, 42, 205-214.

Panum, P. L. (1940). Physiological investigations concerning vision with two eyes (C. Hubscher, Trans.). Hanover, NH: Dartmouth Eye Institute. (Original work published 1858)

RICHARDS, W. (1971). Anomalous stereoscopic depth perception. Journal of the Optical Society of America, 61, 410-414.

ShImojo, S., \& NAKaYAMa, K. (1990). Real world occlusion constraints and binocular rivalry. Vision Research, 30, 69-80.

SHIMONO, K. (1993). Fukuso-undo to ryogansutereopusisu [Effects of vergence movements on binocular stereopsis]. Japanese Journal of Optics, 23, 17-22.

Shimono, K., NaKamizo, S., \& IDA, T. (1994a). Occlusion provides depth-order information but camouflage does not in the WheatstonePanum limiting case. Perception, 23 (Suppl.), 39.

Shimono, K., NaKamizo, S., \& IDa, T. (1994b). Wheatstone-Panum limiting case niokeru okuyukihoukou to ryou no chikaku [The magnitude and direction of perceived depth in the Wheatstone-Panum limiting case]. Vision, 6, 107-110.

Shimono, K., ONo, H., SAIDA, S., \& MAPP, A. (1998). Methodological caveats for monitoring binocular eye position with Nonius stimuli. Vision Research, 38, 591-600.

TAM, W. J., \& ONO, H. (1987). Zero horizontal disparity in binocular depth mixture stimuli. Vision Research, 27, 1207-1210.

WhEATSTONE, C. (1838). Contributions to the physiology of vision: Part the first. On some remarkable, and hitherto unobserved, phenomena of binocular vision. Philosophical Transactions of the Royal Society, 128, 371-394.

\section{NOTES}

1. We use this term to refer to the stimulus arrangement used by Wheatstone (1838) and by Panum (1858/1940). In Wheatstone's stereogram (1838, his Figure 23), different-sized images were used; a thin vertical line intersecting a thick inclined line were presented on one retina and a thick vertical line on the other. On the other hand, in Panum's stereogram (1858/1940, his Figure 53), equal-sized images were used; two vertical lines were presented on one retina and a single vertical line on the other. The important elements of the configuration are that two stimuli are projected onto one retina and a single stimulus is projected onto the other. Our use of the term is consistent with its use by others (e.g., Arditi, 1986; Howard \& Ohmi, 1992; Howard \& Rogers, 1995; Krol, 1982). For a more detailed discussion of what constitutes the Wheatstone-Panum limiting case, refer to Ono, Shimono, and Shibuta (1992)

2. Note that stimulus arrangements of the stimuli in the occlusion and camouflage configurations here are the same as those labeled as locationdefined and location-not-defined conditions, respectively, in Experiment 1 of Ono et al. (1992).

3. Pilot studies indicated that the present method provides a useful and robust index of perceived depth. Results from the pilot studies confirmed that, although there were large individual differences in terms of the absolute values, magnitude of depth estimated by each individual covaried linearly with the range of binocular disparities that were used in our experiments. The observed linear function of perceived depth is consistent with that seen in the results obtained with the matching method (e.g., Richards, 1971) and with the ratio judgment method (e.g., Foley \& Richards, 1972).

(Manuscript received October 13, 1997; revision accepted for publication February 24, 1998.) 